\title{
CORRECTION
}

\section{Correction to: MAP2K6 remodels chromatin and facilitates reprogramming by activating Gatad2b-phosphorylation dependent heterochromatin loosening}

Guangsuo Xing, Zichao Liu, Luyuan Huang, Danyun Zhao (D), Tao Wang, Hao Yuan (iD, Yi Wu (D), Linpeng Li, Qi Long (iD, Yanshuang Zhou, Zhihong Hao (D), Yang Liu, Jianghuan Lu, Shiting Li, Jieying Zhu, Bo Wang, Junwei Wang, Jing Liu, Jiekai Chen, Duanqing Pei (iD, Xingguo Liu (iD) and Keshi Chen (iD

(c) The Author(s), under exclusive licence to ADMC Associazione Differenziamento e Morte Cellulare 2021

Cell Death \& Differentiation (2022) 29:2333; https://doi.org/10.1038/s41418-021-00923-8

Correction to: Cell Death Differ https://doi.org/10.1038/s41418-02100902-z, published online 24 November 2021
The original version of this article unfortunately contained a mistake in the funding. The funding number "2019YFA09004500" should be "2019YFA0904500". The authors apologize for the mistake. The original article has been corrected. 